\title{
Ovarian Yolk Sac Tumor
}

National Cancer Institute

\section{Source}

National Cancer Institute. Ovarian Yolk Sac Tumor. NCI Thesaurus. Code C8107.

A usually rapidly growing malignant germ cell tumor arising from the ovary. It usually occurs in children and adolescents. Signs and symptoms include abdominal pain and a large abdominal or pelvic mass. The serum alpha-fetoprotein is almost always elevated preoperatively. Morphologically, there is marked heterogeneity due to numerous patterns of differentiation coexisting in the same tumor. The most common pattern is reticular. 